\title{
Active sound radiation control with secondary sources at the edge of the opening
}

Shuping Wang ${ }^{\mathrm{a}}$, Jingxia Yu ${ }^{\mathrm{a}}$, Xiaojun Qiu ${ }^{\mathrm{b}}$, Marek Pawelczyk ${ }^{\mathrm{c}}$, Abu Shaid ${ }^{\mathrm{d}}$ and Lijing Wang $^{\mathrm{d}}$

${ }^{\text {a }}$ Institute of Acoustics, Nanjing University, Nanjing, China

${ }^{\mathrm{b}}$ School of Electrical and Computer Engineering and the Design Research Institute, RMIT University, Melbourne, Australia

${ }^{\mathrm{c}}$ Institute of Automatic Control, Silesian University of Technology, Gliwice, Poland

${ }^{\mathrm{d}}$ School of Fashion and Textiles, RMIT University, Melbourne, Australia 


\begin{abstract}
Planar virtual sound barriers with secondary sources over the entire opening have been demonstrated an effective way to achieve global control of sound transmission through the opening, but loudspeakers in the middle of the opening affect ventilation, lighting and normal access through it. To avoid this problem, this technical note proposes to implement secondary sources at the edge of a cavity opening and investigates the active sound reduction performance of the system numerically and experimentally. Unlike secondary sources over the entire opening which can achieve sound reduction at any frequency as long as there are sufficient of them, there exists an upper bound of effective frequency for global control when secondary sources are at the edge of the opening; however, local control is always achievable. Preliminary active noise control experiments were conducted with an open wooden box and in a semi-closed open ceiling meeting room to support the conclusions.
\end{abstract}

Keywords: open cavity; edge; active noise control 


\section{Introduction}

Openings are often used for ventilation and lighting of buildings, but they reduce sound transmission loss of the building facades. Many researchers have worked on different approaches to attenuate sound radiation from openings. For example, quarter-wave resonators and transparent micro-perforated absorbers have been used to reduce noise radiation through openings [1-2]. Maillard and Carter studied passive and active control on openings and found that passive control approaches can improve the sound insulation in mid-high frequency range, while at low frequencies active control systems are more appropriate [3]. De Salis et al. reviewed various noise control techniques for natural ventilation openings and suggested using hybrid systems to achieve broadband sound reduction [4].

Active control strategies have been investigated in previous work. Ise implemented 16 independent single-channel active noise control systems over an entire open window in experiments and achieved a sound reduction of $10 \mathrm{~dB}$ for 200-700 Hz at the error microphones [5]. Huang et al. applied active noise control in a staggered window system and studied its performance by numerical simulations and experiments [6]. Nishimura et al. installed 4 Active Acoustic Shielding cells on a 250 $\mathrm{mm} \times 250 \mathrm{~mm}$ window and the maximum sound reduction achieved at the error microphones was $10 \mathrm{~dB}$ from $500 \mathrm{~Hz}$ to $2000 \mathrm{~Hz}$ [7].

Wang et al. demonstrated that sound power radiated through an opening can be reduced by secondary sources distributed over the entire opening [8]. It was verified experimentally that noise below $500 \mathrm{~Hz}$ can be effectively reduced with 6 secondary sources at a $0.432 \mathrm{~m} \times 0.67 \mathrm{~m}$ opening [9]. However, secondary sources located in the middle of the opening are difficult to implement in some applications. Applying secondary sources only at the edge of the opening is sometimes a more practical way, but its feasibility of achieving global control is not known.

If global sound control is difficult to achieve or unnecessary, local control provides another option [11]. Guo and Pan conducted research on the quiet zones in free space created by multiple secondary sources and error microphones located in two parallel planes, and found that there existed a range of optimal spacing for the 
control sources and error microphones [12]. David and Elliott found that the diameter of $10 \mathrm{~dB}$ quiet zone can be up to about one tenth of the acoustic wavelength [13].

Zou et al. proposed a 16-channel virtual sound barrier (VSB) system which created a cylindrical $10 \mathrm{~dB}$ quiet zone with $0.2 \mathrm{~m}$ height and $0.2 \mathrm{~m}$ radius [15]. Epain and Friot developed an active control system by using the boundary pressure control (BPC) technique with 30 secondary sources and 30 error microphones and created a quiet zone inside the sphere consisting of error microphones [16]. In practical applications, the virtual error sensor arrangement can be applied to avoid the interference problem between the occupant's movements and error microphones [17-20]. The microphones do not need to be an obstacle for the users because the two-stage idea of Virtual Microphone Control can be applied, which only marginally limits the performance of the control system [21].

This note investigates the performance of active control with secondary sources only at the edge of an opening by numerical simulations first. The effective global control frequency is explored and compared with the case when the secondary sources are distributed over the entire opening. Experiments are carried out with an open wooden box and in the Fabpod, a semi-closed meeting room, to explore the possibility of achieving local control with secondary sources at the edge of the opening.

\section{Theory}

In global control, the cost function is defined as the sound power plus the weighted control power to optimize the strengths of secondary sources and improve the stability of the control system

$$
J=\frac{1}{2}\left\{\operatorname{Re}\left[q_{\mathrm{p}}^{\mathrm{H}} p_{\mathrm{p}}\right]+\operatorname{Re}\left[\boldsymbol{q}_{\mathrm{s}}^{\mathrm{H}} \boldsymbol{p}_{\mathrm{s}}\right]\right\}+\beta \boldsymbol{q}_{\mathrm{s}}^{\mathrm{H}} \boldsymbol{q}_{\mathrm{s}},
$$

where $q_{\mathrm{p}}$ is the strength of the primary source; $\boldsymbol{q}_{\mathrm{s}}$ is the vector of the strengths of secondary sources; $p_{\mathrm{p}}$ is the sound pressure at the position of the primary source and $\boldsymbol{p}_{\mathrm{s}}$ is the vector of the sound pressure at positions of secondary sources. $\beta$ is a positive real number to constrain the control effort [22]. The transcript $\mathrm{H}$ denotes a Hermitian transpose and $\operatorname{Re}[$ ] means the real part of the quantity in square brackets. By 
minimizing the cost function in Eq. (1), the optimized strengths of secondary sources can be obtained [23]

$$
\boldsymbol{q}_{\mathrm{s}}=-\left(\boldsymbol{R}_{\mathrm{ss}}+\beta \mathbf{I}\right)^{-1} \boldsymbol{R}_{\mathrm{sp}} q_{\mathrm{p}},
$$

where $\boldsymbol{R}_{\mathrm{ss}}=\operatorname{Re}\left[\boldsymbol{Z}_{\mathrm{ss}}\right], \boldsymbol{R}_{\mathrm{sp}}=\operatorname{Re}\left[\boldsymbol{Z}_{\mathrm{sp}}\right] . \boldsymbol{Z}_{\mathrm{ss}}$ is the acoustic transfer function matrix between the secondary sources and $\boldsymbol{Z}_{\mathrm{sp}}$ is the acoustic transfer function vector between the primary source and secondary sources. I is an identity matrix.

In local control, the cost function is defined as the sum of the squared sound pressure at $L$ error points plus the weighted control source power

$$
J=\boldsymbol{p}^{\mathrm{H}} \boldsymbol{p}+\beta \boldsymbol{q}_{\mathrm{s}}^{\mathrm{H}} \boldsymbol{q}_{\mathrm{s}},
$$

where $\beta$ is also a positive real number for constraining the control effort. The optimized vector of strengths of the secondary sources is [24]

$$
\boldsymbol{q}_{\mathrm{s}}=-\left(\boldsymbol{Z}_{\mathrm{se}}^{\mathrm{H}} \boldsymbol{Z}_{\mathrm{se}}+\beta \mathbf{I}\right)^{-1} \boldsymbol{Z}_{\mathrm{se}}^{\mathrm{H}} \boldsymbol{Z}_{\mathrm{e}} q_{p},
$$

where $\boldsymbol{Z}_{\mathrm{se}}$ is the acoustic transfer function matrix between the secondary sources and error points and $\boldsymbol{Z}_{\mathrm{e}}$ is the acoustic transfer function vector between the primary source and error points.

\section{Simulations and Discussions}

A simple rectangular open cavity in Fig. 1(a) was used as the model for the research. The dimension of the cavity is $1.20 \mathrm{~m}$ (length) $\times 1.00 \mathrm{~m}$ (width) $\times 1.30 \mathrm{~m}$ (height) and all the 5 walls are rigid. It is assumed that the insertion loss of the side walls is sufficiently large so that sound outside the cavity is solely that transmitted through the opening. As it is difficult to obtain the sound field in and outside an unbaffled open cavity analytically [25], a rectangular cavity with its opening embedded at an infinite rigid baffle is investigated here with the method proposed in Ref [9]. 


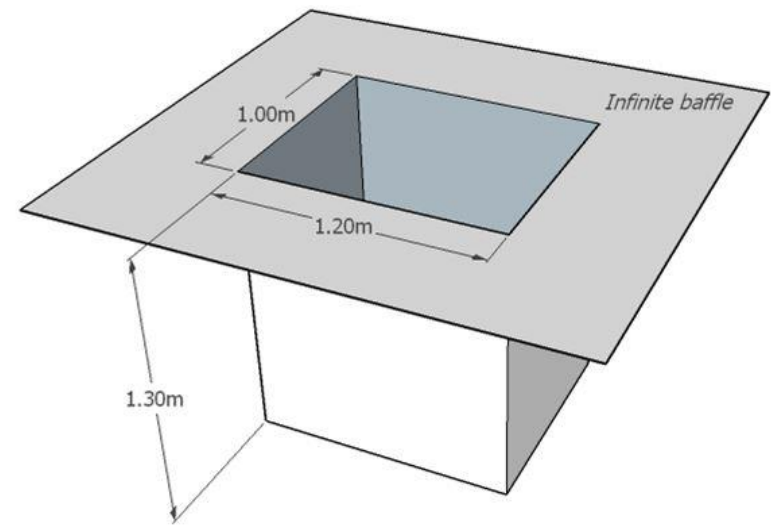

(a)

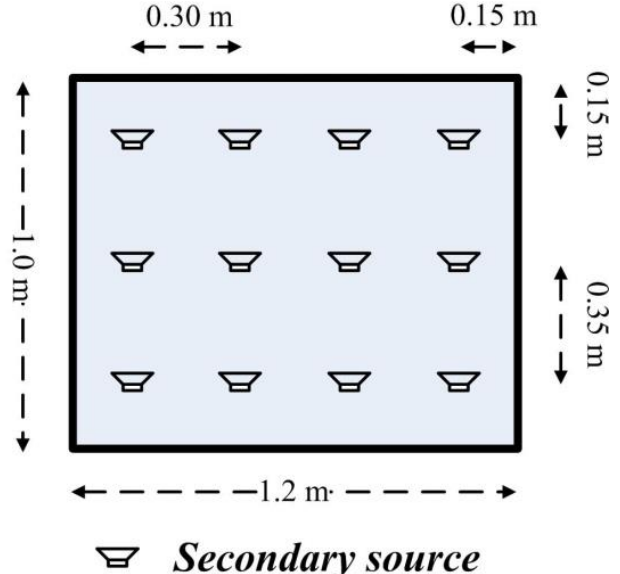

(b)

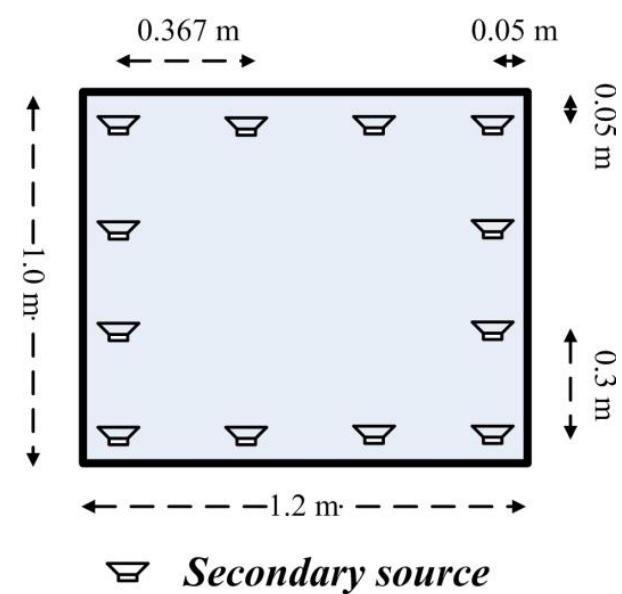

(c)

Fig. 1. (a) The model of the baffled open cavity, (b) Positions of 12 secondary sources evenly distributed over the entire opening, (c) Positions of 12 secondary sources at the edge of the opening.

\subsection{Global control}

The positions of 12 secondary sources evenly distributed over the entire opening are indicated in Fig. 1(b). The intervals in $x$ and $y$ direction are $0.30 \mathrm{~m}$ and $0.35 \mathrm{~m}$, respectively. For the convenience of implementation, 12 secondary sources are fixed inside the cavity near the opening, at the height of $1.25 \mathrm{~m}$. The positions of 12 secondary sources at the edge of the opening are shown in Fig. 1(c). Figure 2 shows the sound power level (SWL) without (Anc off) and with (Anc on) control when 12 secondary sources are evenly distributed over the entire opening and only at the edge. Eq. (2) is used to optimize the strengths of secondary sources and $\beta=0.1$. The tonal primary source is located at $(0.20,0.50,0.80) \mathrm{m}$. 


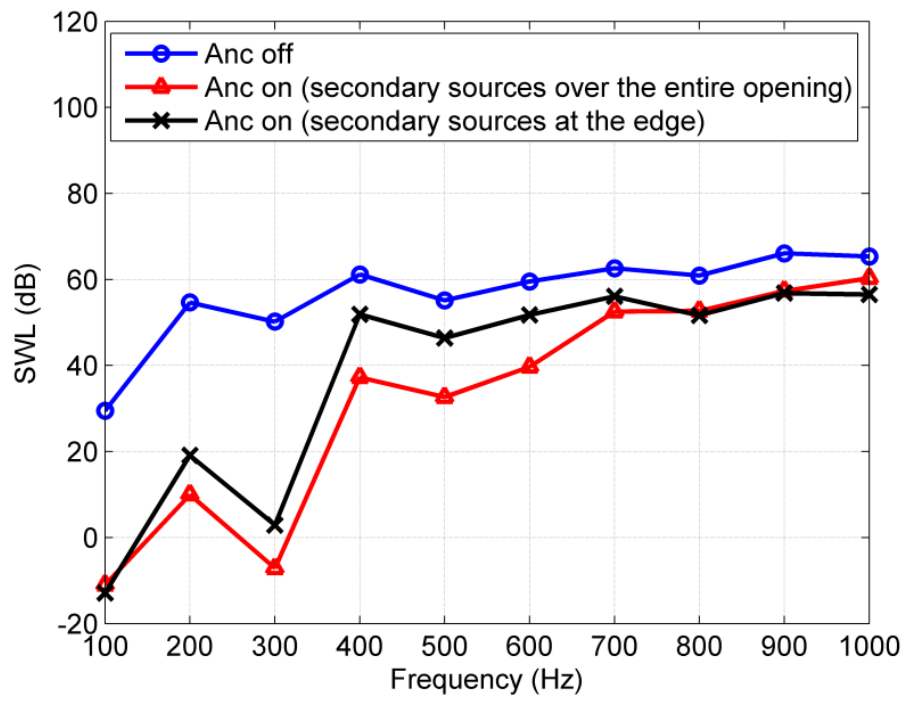

Fig. 2. Sound power level without and with control when secondary sources are distributed over the entire opening and at the edge of the opening.

Figure 2 shows that at frequencies below $300 \mathrm{~Hz}$, both configurations are effective as the sound reductions are more than $30 \mathrm{~dB}$. The sound reduction performance becomes worse as the frequency increases, but the sound reduction from $400 \mathrm{~Hz}$ to $600 \mathrm{~Hz}$ is still more than $20 \mathrm{~dB}$ when the secondary sources are distributed over the entire opening. However, the sound reduction decreases to less than $10 \mathrm{~dB}$ from $400 \mathrm{~Hz}$ to $600 \mathrm{~Hz}$ when the secondary sources are distributed at the edge. It is clear that the ANC system is still effective from $400 \mathrm{~Hz}$ to $600 \mathrm{~Hz}$ when the secondary sources are distributed over the entire opening but the one with secondary sources at the edge is not. At frequencies higher than $600 \mathrm{~Hz}$, both configurations are not effective any more. Therefore, there exist an upper effective frequency for both configurations, and the upper bound is lower when secondary sources are at the edge.

If sufficient secondary sources are distributed over the entire opening, sound at higher frequencies can be attenuated. However, this is not the case when they are distributed only at the edge of the opening. Figure 3 shows the sound power level without and with active control when 12, 24, 36 and 48 secondary sources are distributed at the height of $1.25 \mathrm{~m}$. 


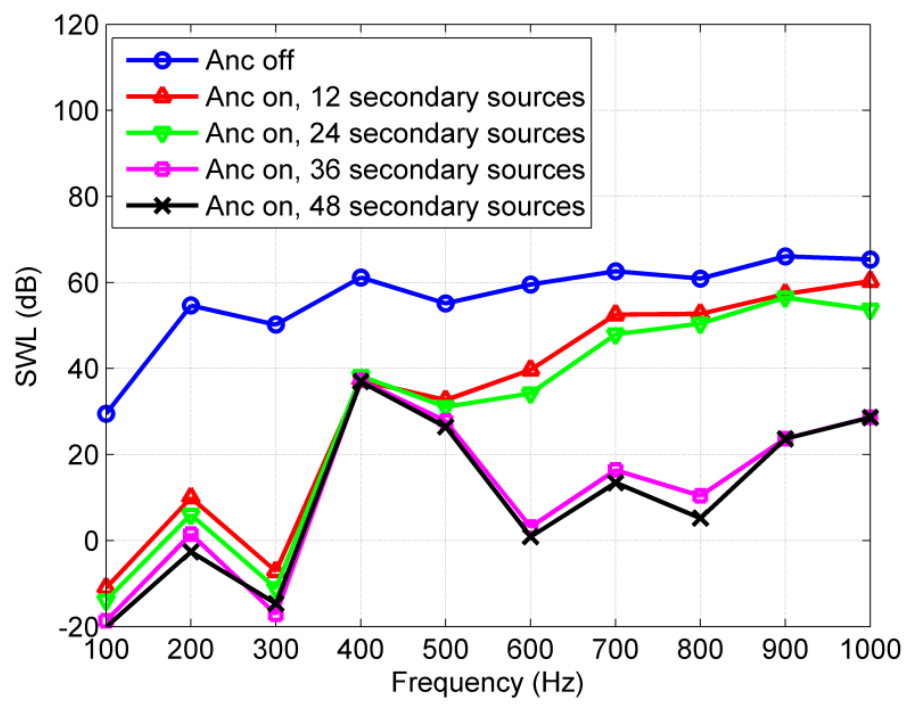

(a)

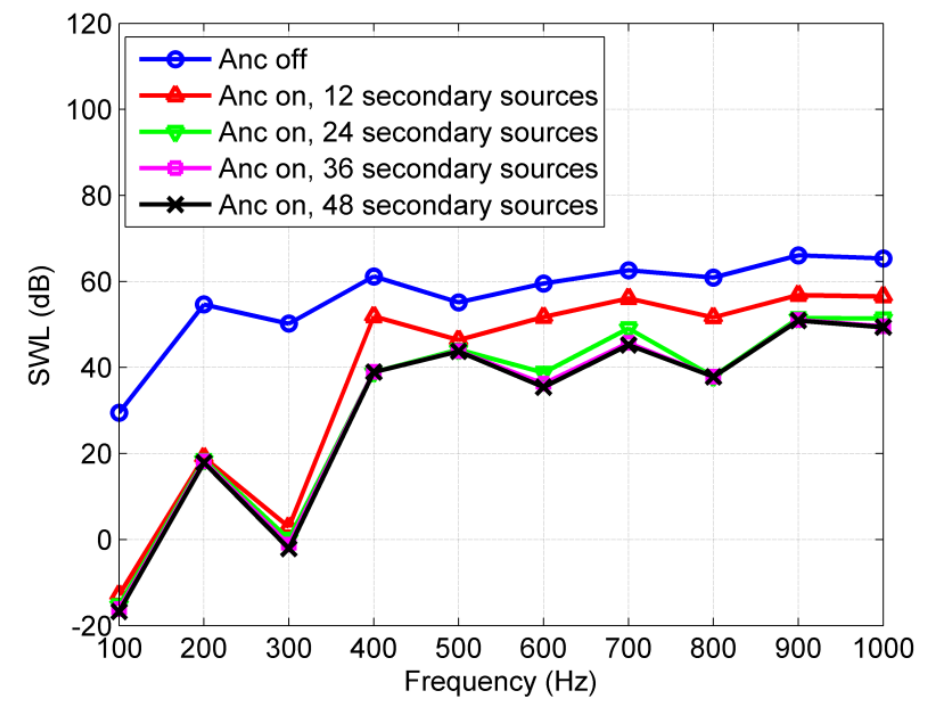

(b)

Fig. 3. The sound power level without and with control with different number of secondary sources, (a) secondary sources are distributed over the entire opening, (b) secondary sources are distributed at the edge of the opening.

Figure 3(a) shows that when 36 or 48 secondary sources are distributed over the entire opening, sound power reduction at $700 \mathrm{~Hz}$ or higher frequencies is significantly higher than 12 or 24 secondary sources. Therefore, more secondary sources lead to effective sound reduction over a wider frequency range when they are distributed over the entire opening. However, in Fig. 3(b), the curves corresponding to 36 and 48 secondary sources are almost the same. This indicates that it is already the best 
performance that can be achieved by the configuration and no extra sound reduction can be obtained even though more secondary sources are applied. The upper bound of effective frequency is about $300 \mathrm{~Hz}$ when secondary sources are distributed at the edge of the opening. The upper bound of effective frequency and the number of secondary sources need to achieve the best noise reduction performance are related to the size of the opening and the cavity, but the relationship is not clear at present and needs to be investigated in the future research.

\subsection{Local control}

In open ceiling meeting rooms, sound radiated to the space around the meeting room is more important (due to neighboring work stations) than that radiated to the upper space of the opening. The feasibility of local control of sound radiation to the space around the open cavity with secondary sources are distributed at the edge of the opening is explored. 16 error points marked by 'o' are set around the open cavity with a perpendicular distance of $1.0 \mathrm{~m}$ between the cavity wall and the error points, shown as Group 1 in Fig. 4.

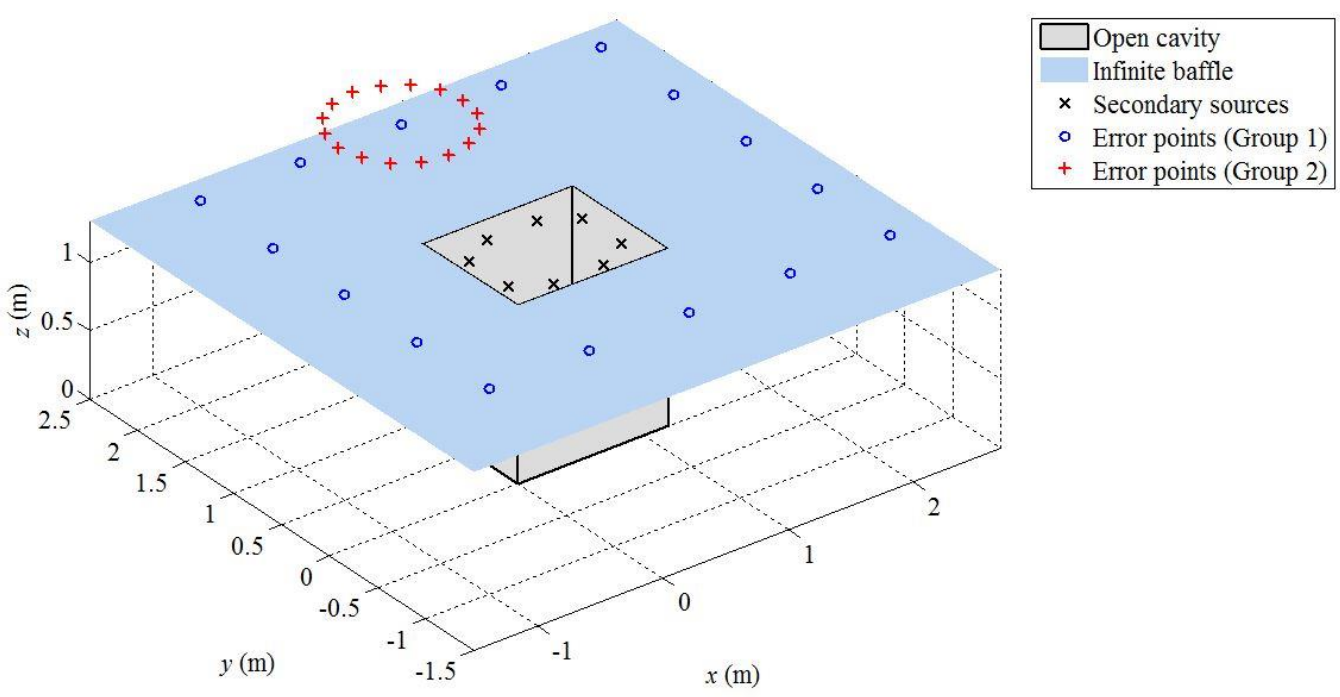

Fig. 4. The physical configuration of the system: positions of secondary sources and error points.

The positions of 8 secondary sources are shown in Fig. 4 marked by ' $x$ '. Two secondary sources $0.05 \mathrm{~m}$ from the boundary are distributed along each side of the opening, and the interval between them is $0.40 \mathrm{~m}$. The sound reduction is defined as 
the difference of the average sound pressure level at 16 error points without and with control. The average sound pressure level at 16 error points without and with control and the corresponding sound reduction are shown in Fig. 5(a). $\beta$ in Eq. (3) is set as 0.1 to constrain the strengths of secondary sources.

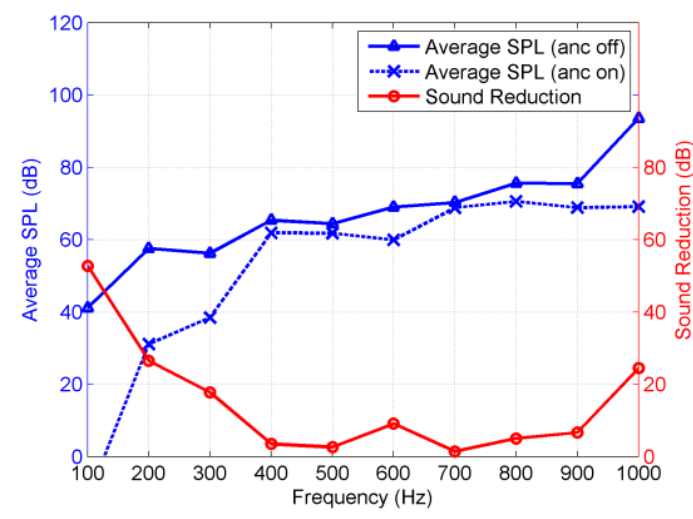

(a)

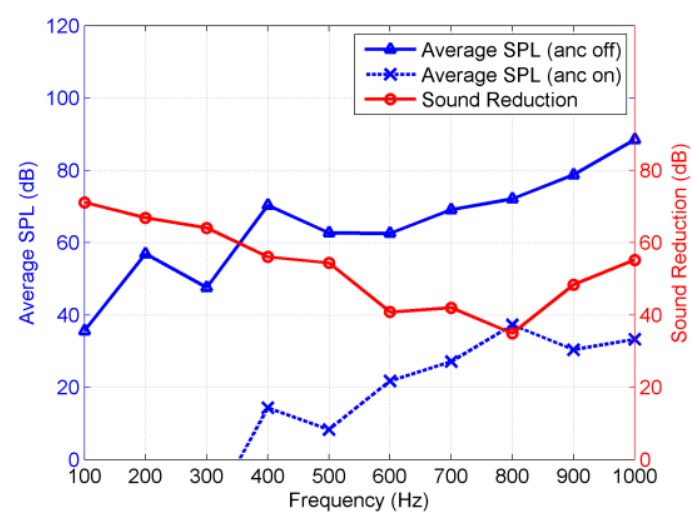

(b)

Fig. 5. The average sound pressure level at error points without and with control and the sound reduction, (a) the error points are Group 1 in Fig. 3, (b) the error points are Group 2 in Fig. 3

In Fig. 5(a), the sound reduction of average sound pressure level becomes negligible from $400 \mathrm{~Hz}$, so local active control is only effective at low frequencies (lower than $300 \mathrm{~Hz}$ ). In Fig. 5(b), the sound reduction at all the frequencies below $1000 \mathrm{~Hz}$ is more than $34 \mathrm{~dB}$ because of the relatively small target area. It might be practical to create a small local quiet zone when secondary sources are implemented at the edge of openings.

\section{Experiments}

\subsection{Experiments with an open wooden box}

An open wooden box made of medium density fiberboard was used as a simplified model of the open cavity. The box has the dimensions of $1.2 \mathrm{~m}$ (length), 1.0 $\mathrm{m}$ (width) and $1.3 \mathrm{~m}$ (height). An 8-channel active noise control system was implemented at the edge of its opening. For the convenience of implementation, 8 secondary sources were fixed outside the wooden box on a frame at the top of the opening, 2 on each side with an interval of $0.4 \mathrm{~m}$. Eight error microphones were placed $0.2 \mathrm{~m}$ away from the outer wall of the wooden box at the height of $1.25 \mathrm{~m}$. The 
experimental setup is shown in Fig. 6(a). Figure 6(b) is a schematic diagram of the physical configurations.

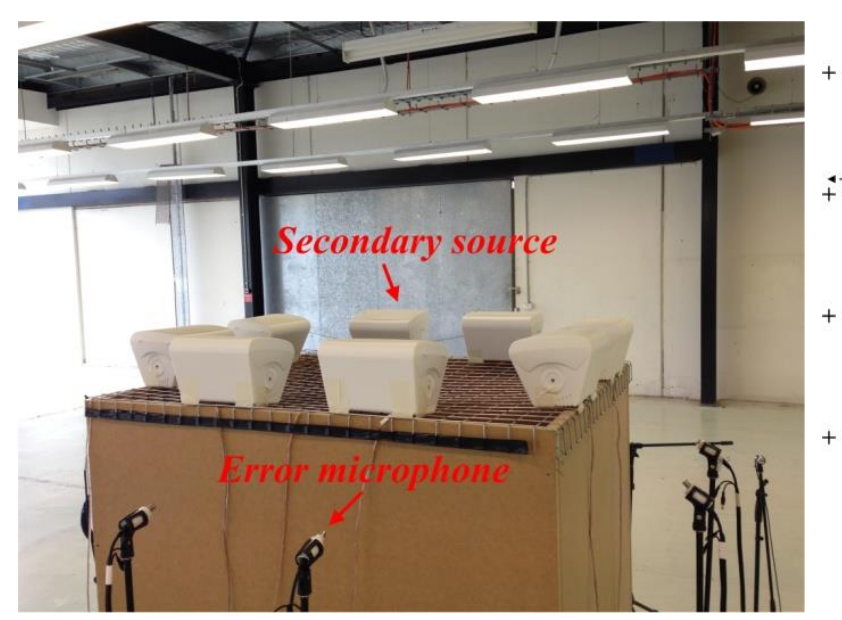

(a)

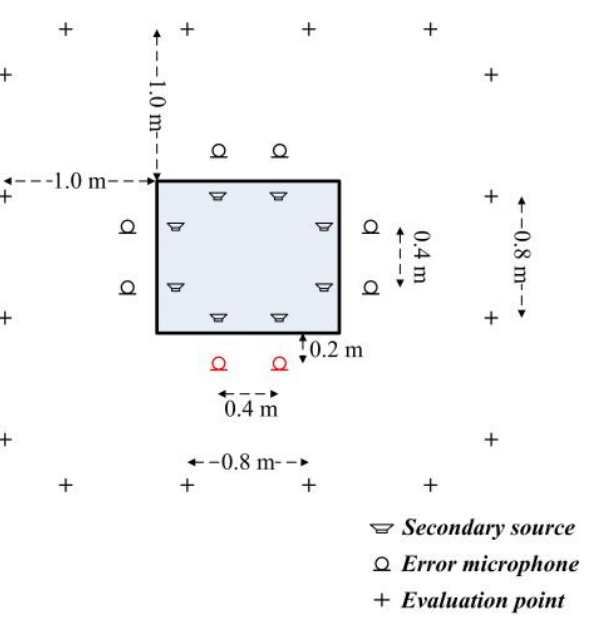

(b)

Fig. 6. The experimental setup, (a) the picture of the experimental setup, (b) schematic diagram of the physical configurations.

The primary source was a loudspeaker inside the wooden box, and a commercial active noise controller (Tiger ANC II) embedded with the fully coupled FxLMS algorithm was used in the experiments. The sampling frequency was $2 \mathrm{kHz}$ and the lengths of the secondary path model and control filters were both 320 taps. The input broadband signal (below $1000 \mathrm{~Hz}$ ) to the primary source was used as the reference signal. The average error signal at 8 error microphones without and with control and the corresponding sound reduction are shown in Fig. 7(a), where that error signal at most frequencies below $1000 \mathrm{~Hz}$ can be successfully attenuated by $5 \mathrm{~dB}$.

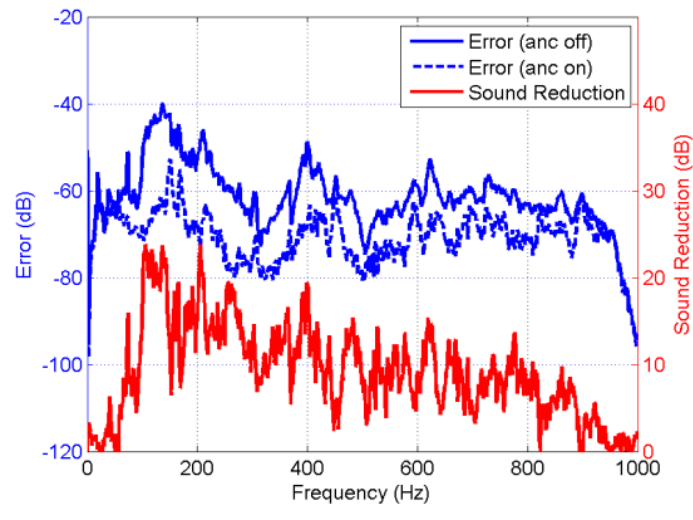

(a)

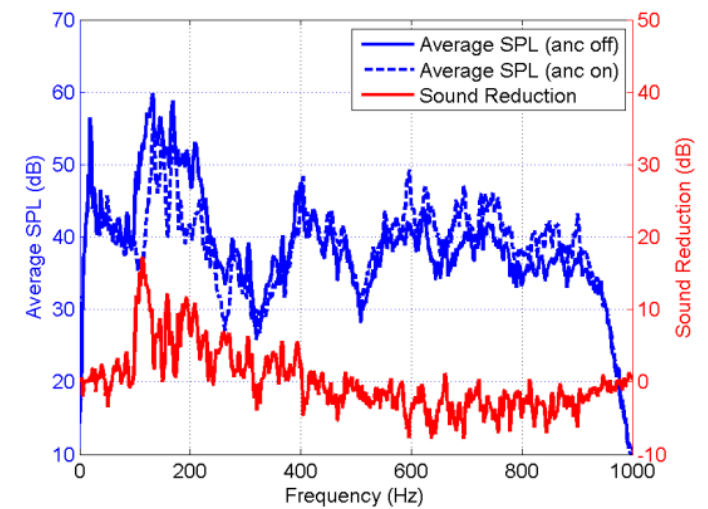

(b)

Fig. 7. (a) Average error signal without and with control and the corresponding sound reduction, 
(b) Average sound pressure level at 16 evaluation points without and with control and the corresponding sound reduction.

To evaluate the sound reduction performance at other locations around the wooden box, the sound pressure levels without and with control at 16 evaluation points $1.0 \mathrm{~m}$ away from the wooden box (shown in Fig. 6(b)) were measured and the average sound pressure level and sound reduction are shown in Fig. 7(b). In Fig. 7(b), the average sound reduction at 16 evaluation points is $8.7 \mathrm{~dB}$ at $100 \mathrm{~Hz}$ and $6.8 \mathrm{~dB}$ at $200 \mathrm{~Hz}$, and there is no sound reduction at frequencies higher than $400 \mathrm{~Hz}$. The upper bound of effective frequency is about $400 \mathrm{~Hz}$.

To assess the size of effective sound reduction area around the error points, the sound pressure level at 2 points about the same distance away from an error point and 2 points about the same distance away from another error point is measured. It is found that the sound reduction decreases with the distance from the error points. The effective $10 \mathrm{~dB}$ sound reduction area is about $0.2 \mathrm{~m}$ around the error points below 400 Hz. Within the effective sound reduction area (no more than $0.2 \mathrm{~m}$ from the error points), the sound reduction is higher at low frequencies than at relatively high frequencies.

As the sum of squared sound pressure at error points is the cost function, sound reduction is the maximum at error points. The farther the evaluation point is away from the error points, the less the sound reduction will be. The reason why the sound pressure level in the region of the evaluation points can be reduced by minimizing the sound pressure at error sensors is because of the continuous properties of sound field and/or mapping from the sound field at error sensors to that at the evaluation points. The sound reduction performance at evaluation points can be improved by optimizing the number and positions of error microphones or using different cost function for minimization. It should be noted that the sound reduction performance at evaluation points depends on the acoustical properties of the room as well; however, the relationship is not simple or straightforward. 


\subsection{Experiments in the Fabpod}

Fabpod is a semi-closed meeting room located in an open plan office in the Design Research Institute of RMIT University. It has highly articulated surface composed of hyperboloid cells with different materials, as shown in Fig. 8(a). It has 2 main openings, one is the open ceiling and the other is the entrance. An 8-channel active noise control system was implemented at the open ceiling to investigate the feasibility of achieving local control with the secondary sources at the edge of the opening. Figure 9(b) shows the physical configuration of the system. 8 secondary sources are evenly distributed at one of the edges of the Fabpod and the interval between each other is about $0.40 \mathrm{~m} .8$ error microphones are placed around a chair, which is about $3.0 \mathrm{~m}$ away from the Fabpod. They are at the height of $1.20 \mathrm{~m}$ and approximately evenly distributed on a circle with a radius of $0.70 \mathrm{~m}$.

The objective of this active control experiment was to reduce sound radiation from inside the Fabpod to the space around the chair. The sound source was a semi-omni directional loudspeaker installed inside the Fabpod. Figure 9(c) is a picture of secondary sources and Fig. 9(d) is the picture of error microphones around the chair on a circle with a radius of about $0.35 \mathrm{~m}$.

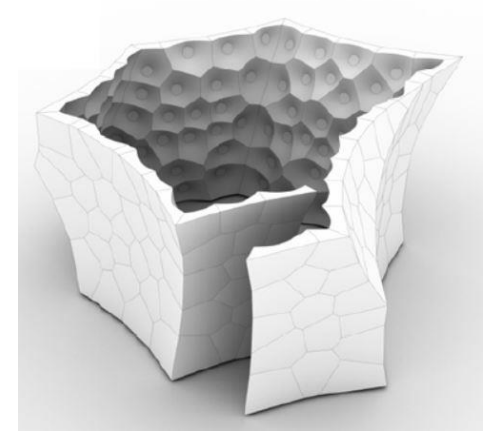

(a)

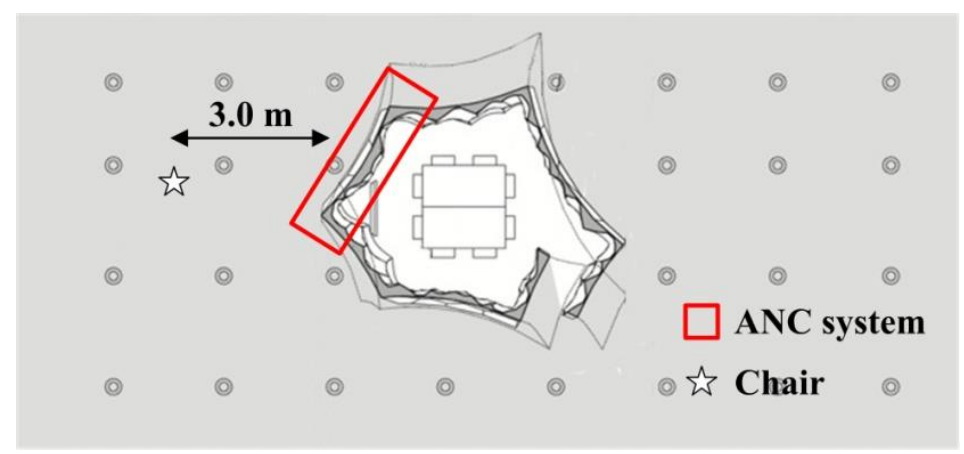

(b) 


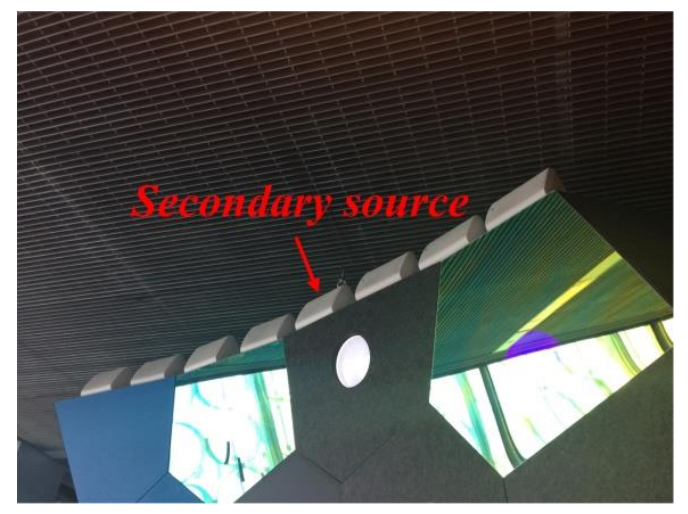

(c)

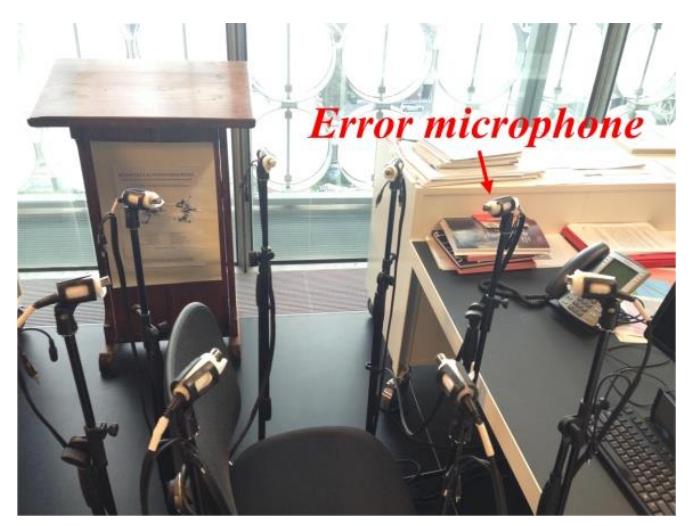

(d)

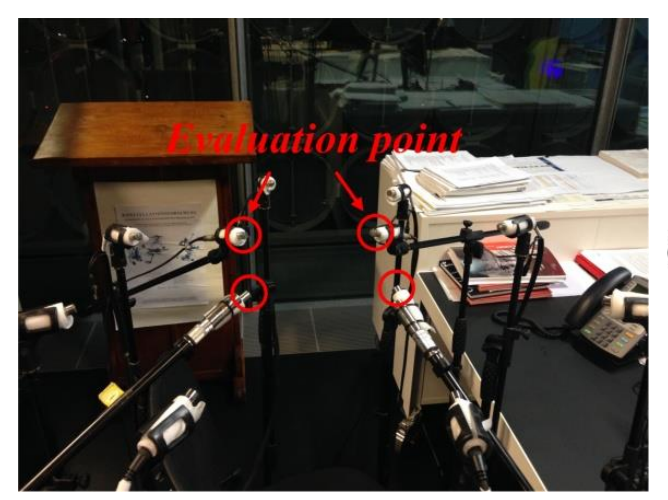

(e)

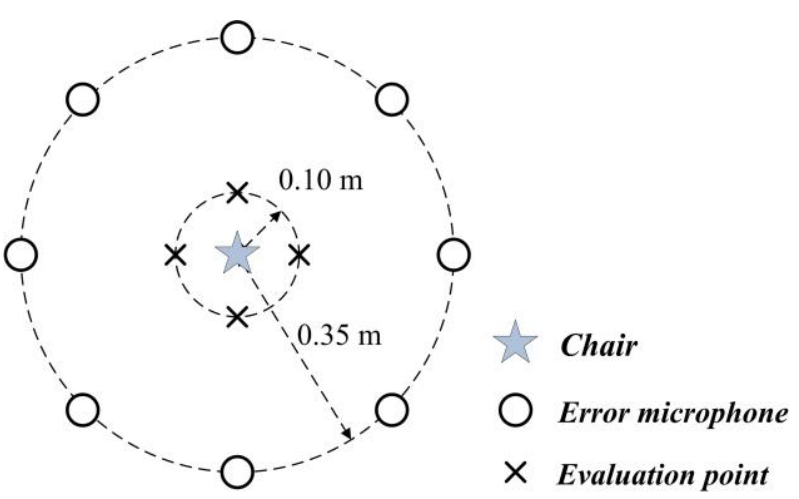

(f)

Fig. 8. The experimental setup of the active noise control system in the Fabpod, (a) the Fabpod, (b) schematic diagram of the physical configurations, (c) 8 secondary sources, (d) 8 error microphones, (e) 4 evaluation points, (f) schematic diagram of the positions of 8 error microphones and 4 evaluation points.

During the experiment, the primary source generated broadband noise below $1000 \mathrm{~Hz}$ and it was also applied as the reference signal. The average error signal without and with control and the corresponding sound reduction are shown in Fig. 9(a), where the sound reduction at most frequencies is more than $5 \mathrm{~dB}$. At frequencies around $150 \mathrm{~Hz}, 190 \mathrm{~Hz}, 250 \mathrm{~Hz}, 690 \mathrm{~Hz}, 720 \mathrm{~Hz}$ and $810 \mathrm{~Hz}$, the sound reduction is more than $10 \mathrm{~dB}$ because of the relatively high primary sound pressure level. The overall sound reduction is $9.9 \mathrm{~dB}$ for broadband noise below $1000 \mathrm{~Hz}$. 


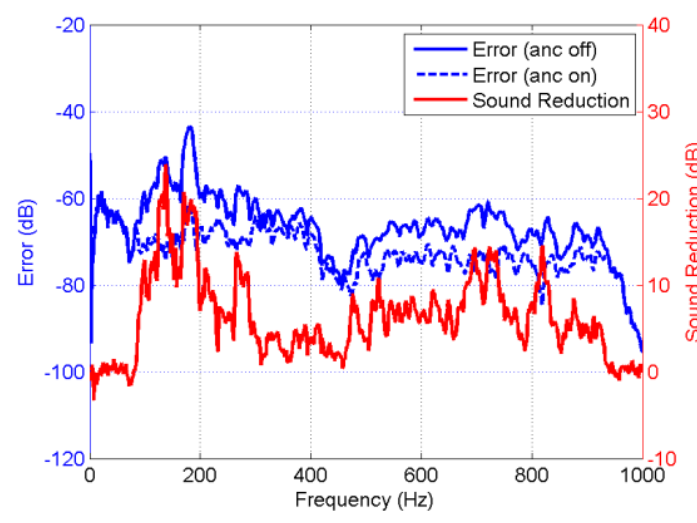

(a)

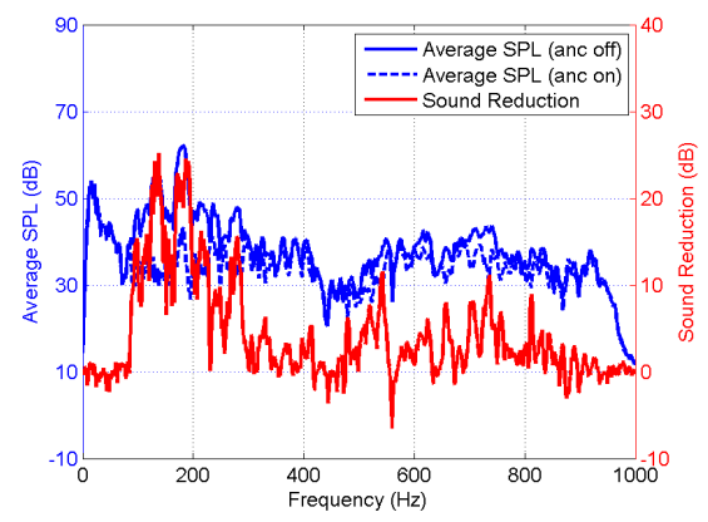

(b)

Fig. 9. (a) The average error signal without and with control and the sound reduction, (b) The average sound pressure level at 4 evaluation points without and with control and the sound reduction.

To evaluate the sound reduction performance at other points within the local area, the sound pressure level at 4 evaluation points inside the circle consisting of error microphones was measured. The positions of these points are shown in Fig. 9(e-f). The measured results in Fig. 10(b) show that the system created a quiet zone with a radius of about $0.35 \mathrm{~m}$ within which sound between $100 \mathrm{~Hz}$ and $280 \mathrm{~Hz}$ is reduced by $10 \mathrm{~dB}$ and the overall sound reduction for broadband noise is $8.6 \mathrm{~dB}$.

The system does not have good performance below $100 \mathrm{~Hz}$ because both the primary and secondary sources do not have the capability to generate large sound at very low frequencies. The reason that the performance is poor at frequencies above $280 \mathrm{~Hz}$ is because a quarter of the wavelength is less than $0.3 \mathrm{~m}$, which is shorter than the distance between the error sensor and evaluation point, so cancelling the primary sound at error sensors cannot guarantee the reduction of sound pressure level at the evaluation points due to the continuity of sound field.

\section{Conclusions}

This note proposes to implement secondary sources at the edge of a cavity opening to achieve active control of sound radiation from inside the cavity to outside. Numerical simulations based on a simplified model of a baffled open cavity demonstrate that there exists an upper bound frequency for effective global control 
when secondary sources are installed at the edge of the opening, but local control to create a quiet zone is always feasible. Preliminary active noise control experiments were conducted with an open wooden box and in a semi-closed open ceiling meeting room to support the conclusions.

\section{Acknowledgements}

This research was supported under Australian Research Council's Linkage Projects funding scheme (LP140100740) and an internal project of Design Research Institute of RMIT University. It was also partly supported by The National Science Centre, Poland, decision no. DEC-2012/07/B/ST7/01408.

\section{References}

[1] Field C and Fricke F. Theory and applications of quarter-wave resonators: a prelude to their use for attenuating noise entering buildings through openings. Appl Acoust 1998; 53: 117-32.

[2] Kang $\mathbf{J}$ and Brocklesby M. Feasibility of applying micro-perforated absorbers in acoustic window systems. Appl Acoust 2005; 66: 669-89.

[3] Maillard J, Guigou-Carter C. Study of passive/active control on openings for natural ventilation in buildings. Proceedings of Inter-noise 2000, Nice, France; 2000.

[4] De Salis M, Oldham D, Sharples S. Noise control strategies for naturally ventilated buildings. Building Environ 2002; 37(5): 471-84.

[5] Ise S. The boundary surface control principle and its applications," IEICE Trans. Fundamentals 2005; E88-A: 1656-64.

[6] Huang H, Qiu X, Kang J. Active noise attenuation in ventilation windows. J Acoust Soc Am 2011; 130(1): 176-88.

[7] Nishimura M, Ohnishi K, Kanamori N, Ito K. Basic study on active acoustic shielding. Proceedings of Inter-noise 2008, Shanghai, China; 2008. 
[8] Wang S, Tao J, Qiu X. Active control of transformer noise radiated outside a three-dimensional building with one opening. Proceedings of 21 st International Congress on Sound and Vibration, Beijing, China; 2014.

[9] Wang S, Tao J, Qiu X. Performance of a planar virtual sound barrier at the baffled opening of a rectangular cavity. J Acoust Soc Am 2015; 138(5): 2836-47.

[10] Saha P, Pierce A. Geometrical theory of diffraction by an open rectangular box. J Acoust Soc Am 1984; 75(1): 46-9.

[11] Nelson P, Elliott S. Active Control of Sound. Academic Press; 1992.

[12] Guo J, Pan J. Further investigation on actively created quiet zones by multiple control sources in free space. J Acoust Soc Am 1997; 102(5): 3050-53.

[13] David A, Elliott S. Numerical studies of actively generated quiet zones. Appl Acoust 1994; 41(1): 63-79.

[14] Qiu X, Li N, Chen G. Feasibility study of developing practival virtal sound barrier system. Proceedings of 12th International Congress on Sound and Vibration, Lisbon, Portugal; 2005.

[15] Zou H, Qiu X, Lu J, Niu F. A preliminary experimental study on virtual sound barrier system. J Sound Vib 2007; 307: 379-85.

[16] Epain N, Friot E. Active control of sound inside a sphere via control of the acoustic pressure at the boundary surface. J Sound Vib 2007; 299: 587-604.

[17] Garcia J, Elliott S, Boucher C. Generation of zones of quiet using a virtual microphone arrangement. J Acoust Soc Am 1997; 101(6): 3498-516.

[18] Rafaely B, Elliott, Garcia J. Broadband performance of an active headrest. J Acoust Soc Am 1999; 106(2): 787-93.

[19] Zou H, Qiu X, Li N. A numerical study of virtual sound barrier with virtual sensors. Proceedings of the 44th International Congress \& Exhibition on Noise Control Engineering, San Francisco, California, USA; 2015.

[20] Zou H, Qiu X. Optimization of the locations of the virtual sensors in the virtual sound barrier system. J Nanjing University (Natural Sciences) 2009; 45(1): 57-65.

[21] Pawelczyk M. Adaptive noise control algorithms for active headrest system. Control Engineering Practice 2004; 12: 1101-12. 
[22] Kirkeby O, Nelson P, Orduna-Bustamante F, Hamada H. Local sound field reproduction using digital signal processing. J Acoust Soc Am 1996; 100(3): 1584-93.

[23] Elliott S, Joseph P, Nelson P, Johnson M. Power output minimization and power absorption in the active control of sound. J Acoust Soc Am 1991; 90(5): 2501-12.

[24] Elliott S. Signal processing for active control. Academic Press; 2001.

[25] Levine H, Schwinger J. On the radiation of sound from an unflanged circular pipe. Physical Review 1948; 73: 383-406. 